\title{
Estudo da oxidação de cerâmicas à base de carbeto de silício sinterizado via fase líquida utilizando nitreto de alumínio e óxido de ítrio como aditivos
}

\section{(Study of oxidation in liquid phase sintered silicon carbide with addition of aluminum nitride and yttrium oxide)}

\author{
M. J. Bondioli ${ }^{1}$, C. Santos ${ }^{1}$, K. Strecker ${ }^{2}$ \\ ${ }^{1}$ Escola de Engenharia de Lorena, EEL-USP, Pólo Urbo Industrial, Gleba AI 6, s/n, Lorena, SP 12600-000 \\ ${ }^{2}$ Departamento de Engenharia Mecânica - UFSJ, Praça Frei Orlando 170, S. João del Rei, MG 36307-352 \\ mjbondioli@itelefonica.com.br
}

\begin{abstract}
Resumo
Materiais cerâmicos à base de carbeto de silício foram desenvolvidos através de sinterização via fase líquida usando $\mathrm{AlN}_{\mathrm{N}} \mathrm{Y}_{2} \mathrm{O}_{3}$ como

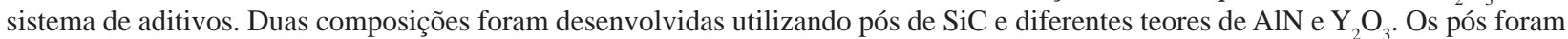
misturados e homogeneizados, secados e subseqüentemente desaglomerados. As misturas do pó foram compactadas por prensagem uniaxial com subseqüente prensagem isostática a frio e os compactos foram sinterizados a $2080{ }^{\circ} \mathrm{C}$, por $1 \mathrm{~h}$, em atmosfera 0,2 $\mathrm{MPa}$ de $\mathrm{N}_{2}$. As amostras sinterizadas foram caracterizadas por difração de raios X e pela sua densidade relativa. O comportamento da oxidação foi investigado e relacionado ao teor de aditivos. Para tanto, as amostras foram submetidas aos ensaios de oxidação em temperaturas de 1200,1300 e $1400{ }^{\circ} \mathrm{C}$, ao ar por 120 h. O ganho de massa das amostras foi traçado em função do tempo de exposição, obtendo a evolução da oxidação na superfície das amostras. A composição das fases cristalinas presentes nas superfícies oxidadas foi obtida utilizando difração de raios X. Baseados nos resultados foram determinados os coeficientes de crescimento parabólico da taxa de oxidação referentes a cada composição estudada. Os resultados indicam que as amostras apresentam oxidação com comportamento parabólico em todas as condições, sendo que as amostras contendo menor quantidade de $\mathrm{Y}_{2} \mathrm{O}_{3}$ em relação ao AlN apresentaram maior resistência a oxidação quando submetidas a temperatura de $1200{ }^{\circ} \mathrm{C}$; porém com o aumento da temperatura para $1400{ }^{\circ} \mathrm{C}$, as amostras contendo maior quantidade de $\mathrm{Y}_{2} \mathrm{O}_{3}$ em relação ao AlN apresentaram maior resistência à oxidação, fato relacionado com as fases intergranulares presentes no sistema, após a sinterização.

Palavras-chave: carbeto de silício, sinterização via fase líquida, oxidação.
\end{abstract}

\begin{abstract}
Silicon carbide ( $\mathrm{SiC}$ ) ceramics were developed by liquid phase sintering using $\mathrm{AlN}-\mathrm{Y}_{2} \mathrm{O}_{3}$ as additive. Two compositions were obtained using different AlN- $\mathrm{Y}_{2} \mathrm{O}_{3}$ contents. The powders were mixed/homogenized and subsequently deagglomerated. Powder mixtures were compacted by cold isostatic pressing and sintered at $2080^{\circ} \mathrm{C}$ for $1 \mathrm{~h}$ under $0.2 \mathrm{MPa}-\mathrm{N}_{2}$ atmosphere. Sintered samples were characterized by X-ray diffraction and relative density. The oxidation behavior was investigated and related to the additive content. Samples were submitted to the tests at 1200, 1300 and $1400{ }^{\circ} \mathrm{C}$ in air for $120 \mathrm{~h}$. Weight gain of the samples was plotted as a function of the exposure time, obtaining the evolution of the oxidation on the sample surfaces. The oxidized surfaces of samples were characterized by X-ray diffraction. The parabolic growing coefficient of the oxidation layer $\left(k_{p}\right)$ were determined. The results indicate that the samples show parabolic behavior in all conditions. Samples with larger $\mathrm{AlN}$ content in relation to $\mathrm{Y}_{2} \mathrm{O}_{3}$ show larger oxidation resistance at $1200{ }^{\circ} \mathrm{C}$; however, with the increase of the temperature to $1400{ }^{\circ} \mathrm{C}$, the samples with smaller AlN content in relation to $\mathrm{Y}_{2} \mathrm{O}_{3}$ show larger oxidation resistance. This fact is related with the intergranular phases present in the system after sintering.
\end{abstract}

Keywords: silicon carbide, liquid phase sintering, oxidation.

\section{INTRODUÇÃO}

O carbeto de silício, $\mathrm{SiC}$, é um material cerâmico sintético que apresenta densidade relativamente baixa, alta dureza, elevada estabilidade térmica e boa condutividade térmica, resultando, dentre outras características, em boa resistência ao desgaste e ao choque térmico, sendo amplamente utilizadas como abrasivos e refratários. Normalmente, o SiC é sinterizado via fase sólida com pequenas adições de $\mathrm{C}, \mathrm{Al}$, $\mathrm{B}$ e seus compostos como por exemplo $\mathrm{B}_{4} \mathrm{C}, \mathrm{Al}_{4} \mathrm{C}$, $\mathrm{AlN}$ etc. [1-3]. Estes tipos de aditivos possuem desvantagens já que são adicionados em pequenas quantidades, em torno de 1 a $2 \%$, o que dificulta a preparação de misturas homogêneas e conseqüentemente a sua homogênea densificação. Um método alternativo à sinterização no estado sólido foi proposto no inicio dos anos 80 [4], com sinterização 
de $\mathrm{SiC}$ com altas densidades relativas, via sinterização por fase líquida, usando misturas de $\mathrm{Al}_{2} \mathrm{O}_{3}$ e $\mathrm{Y}_{2} \mathrm{O}_{3}$ como aditivos. Desde então, nota-se um interesse crescente pelo $\mathrm{SiC}$ sinterizado via fase líquida, haja visto que esse tipo de material oferece a possibilidade de alcançar uma maior tenacidade à fratura baseando-se na alta densificação e no controle da microestrutura [5-8]. Nesses materiais sinterizados via fase líquida, há a formação de uma fase intergranular, normalmente amorfa, entre os grãos de $\mathrm{SiC}$, resultante da reação entre partículas de $\mathrm{SiC}$ e a fase líquida formada pela fusão dos aditivos durante a sinterização. Esta fase amorfa promove um efeito significativamente negativo nas propriedades mecânicas a altas temperaturas, que pode ser contornado através de dois métodos efetivos: o aumento de refratabilidade das fases presentes nos contornos de grãos e a promoção de uma cristalização da fase intergranular amorfa [9]. Devido a possibilidade de aplicação desses materiais em altas temperaturas, o comportamento da oxidação de cerâmicas à base de $\mathrm{SiC}$ sinterizado via fase líquida com a adição de diferentes sistemas de aditivos vem sendo objeto de recentes investigações [9-11]. A resistência à oxidação dos materiais sinterizados via fase líquida é menor do que a dos materiais obtidos por deposição química de vapor (CVD-SiC) ou do material sinterizado por fase sólida com adição de boro, pois contém uma fase de contornos de grãos derivada dos aditivos. Sabe-se que o cátion dos aditivos e das impurezas dos pós de partida reduz a resistência à oxidação de cerâmicos não óxidos tais como $\mathrm{SiC} \mathrm{e} \mathrm{Si}_{3} \mathrm{~N}_{4}$.

Neste trabalho, a resistência a oxidação de materiais cerâmicos a base de SiC aditivado com $\mathrm{AlN} / \mathrm{Y}_{2} \mathrm{O}_{3}$ foi avaliada, comparando-se os efeitos da temperatura na qual os testes foram realizados e a composição dos aditivos utilizada, na resistência a oxidação desses materiais cerâmicos.

\section{MATERIAIS E MÉTODOS}

Foram utilizados como matérias-primas pós de alta pureza a base de $\beta$-SiC (H.C. Starck, BF-12), $\alpha$-SiC (Lonza, UF15), AlN (H.C. Starck, tipo C) e $\mathrm{Y}_{2} \mathrm{O}_{3}$ (H.C. Starck, tipo C), conforme proporções apresentadas na Tabela I.

Tabela I - Composição das misturas de pós (\% em peso) [Table I - Composition of the powder mixtures wt.\%)]

\begin{tabular}{ccccc}
\hline Designação & $\beta$-SiC & $\alpha$-SiC & $\mathrm{AlN}$ & $\mathrm{Y}_{2} \mathrm{O}_{3}$ \\
\hline SY20 & 79 & 1 & 8,42 & 11,58 \\
SY60 & 79 & 1 & 2,16 & 17,84 \\
\hline
\end{tabular}

A homogeneização das misturas foi realizada em moinho tipo atritor via úmido, com álcool isopropílico como veículo, utilizando jarro e bolas de $\mathrm{Si}_{3} \mathrm{~N}_{4}$ sinterizado com velocidade de $1000 \mathrm{rpm}$ durante $4 \mathrm{~h}$. As misturas de pós foram compactadas em dois estágios, prensagem uniaxial a frio (30 MPa - $30 \mathrm{~s}$ ) e subseqüente prensagem isostática a frio, aplicando-se $300 \mathrm{MPa}$ durante $60 \mathrm{~s}$. Compactos com dimensões de aproximadamente $5 \times 20 \times 60 \mathrm{~mm}^{3}$ foram confeccionados.

A sinterização dos corpos-de-prova foi realizada em um forno de resistência de grafite. O ciclo de sinterização utilizado consistiu em aquecimento sob vácuo até $1000{ }^{\circ} \mathrm{C}$ (com taxa de aquecimento $20{ }^{\circ} \mathrm{C} / \mathrm{min}$ ), Nesse instante foi injetado $0,1 \mathrm{MPa}$ de nitrogênio, seguido por um aquecimento até $1600{ }^{\circ} \mathrm{C}$ com uma isoterma de $30 \mathrm{~min}$. Ao final deste estágio, a pressão de $\mathrm{N}_{2}$ foi aumentada para $0,2 \mathrm{MPa}$ e finalmente as amostras foram aquecidas, com uma taxa de aquecimento de $10{ }^{\circ} \mathrm{C} / \mathrm{min}$ até $2080{ }^{\circ} \mathrm{C}$, com um patamar de sinterização de $1 \mathrm{~h}$. Após a sinterização, os corpos-deprova foram retificados e cortados, obtendo-se amostras com dimensões finais $3 \times 4 \times 45 \mathrm{~mm}^{3}$.

Os ensaios de oxidação foram realizados em um forno elétrico horizontal com resistência de $\mathrm{MoSi}_{2}$, com tubo de $\mathrm{Al}_{2} \mathrm{O}_{3}$ acoplado. Dentro desse tubo, foi acondicionado um cadinho de alumina, no interior do qual foram posicionados dois fios finos de uma liga Fe-Cr-Al (Kanthal A1). Os corpos de prova de $\mathrm{SiC}$ foram previamente pesados em balança de precisão e apoiados sobre esses fios de Kanthal A1 sendo, em seguida, submetidos aos testes de oxidação. Os ensaios foram realizados em 1200,1300 e $1400{ }^{\circ} \mathrm{C}$, com um corpode-prova para cada composição e temperatura. Todos os ensaios foram encerrados após $120 \mathrm{~h}$.

Periodicamente (de hora em hora até $8 \mathrm{~h}$, e em seguida a cada $24 \mathrm{~h}$ ) a massa dos corpos-de-prova foi medida, utilizando-se uma balança analítica de alta precisão, avaliando o ganho de massa em função do tempo de exposição das amostras a oxidação. Os resultados de oxidação foram representados graficamente em função do ganho de massa por unidade de área $\left(\mathrm{mg} / \mathrm{cm}^{2}\right) \mathrm{x}$ tempo $(\mathrm{em} \mathrm{h})$, determinando assim o tipo de comportamento de oxidação e as taxas de oxidação dos materiais. $\mathrm{O}$ comportamento à oxidação foi expresso pela equação parabólica de ganho de massa em função do tempo de exposição, através da equação A:

$$
\left(\frac{\Delta \mathrm{m}}{\mathrm{A}_{0}}\right)^{2}=\mathrm{k}_{\mathrm{p}} \mathrm{t}
$$

Onde:

$\frac{\Delta \mathrm{m}}{\mathrm{A}_{0}}=$ ganho de massa por unidade de área $\left(\mathrm{em} \mathrm{mg} / \mathrm{cm}^{2}\right)$;

$\mathrm{k}_{\mathrm{P}}=$ coeficiente de crescimento parabólico da taxa de oxidação $\left(\mathrm{em} \mathrm{mg}^{2} \mathrm{~cm}^{-4} \mathrm{~s}^{-1}\right)$;

$\mathrm{t}=$ tempo de exposição (em segundos).

A densidade das amostras sinterizadas foi determinada pelo método de Arquimedes e os resultados foram correlacionados com a densidade teórica das misturas, visando obter a densidade relativa das cerâmicas sinterizadas.

As fases nas amostras sinterizadas e nas superfícies oxidadas foram identificadas por difração de raios $\mathrm{X}$ em difratômetro Shimadzu XRD6000, com radiação $\mathrm{Cu}-\mathrm{K}_{\alpha}$ $(\lambda=1,5418 \AA$ ) , e com fichas de identificação JCPDS- ICDD, 
foi possível analisar e detectar as fases em cada composição. As condições de execução das análises foram similares para todas as composições, sendo o passo angular $0,05^{\circ} \operatorname{com} 2 \mathrm{~s}$ de tempo por passo.

\section{RESULTADOS E DISCUSSÃO}

Os resultados da massa específica teórica, densidade medida, densidade relativa e perda de massa durante a sinterização, estão listados na Tabela II.

Tabela II - Massa específica teórica $\rho_{\text {th }}$, massa específica após sinterização $\rho_{\mathrm{s}}$, densidade relativa DR e perda de massa $\Delta \mathrm{m}$ das amostras sinterizadas.

[Table II - Theoretical density $\varrho_{t h}$, density after sintering $\rho_{s}$, relative density $D R$ and weight loss $\Delta m$ of the sintered samples. ]

\begin{tabular}{ccccc}
\hline Amostra & $\begin{array}{c}\rho_{\text {th }} \\
{\left[\mathrm{g} / \mathrm{cm}^{3}\right]}\end{array}$ & $\begin{array}{c}\rho_{\mathrm{s}} \\
{\left[\mathrm{g} / \mathrm{cm}^{3}\right]}\end{array}$ & $\begin{array}{c}\text { DR } \\
{[\%]}\end{array}$ & $\begin{array}{c}\Delta \mathrm{m} \\
{[\%]}\end{array}$ \\
\hline SY20 & 3,36 & $3,22 \pm 0,03$ & $95,85 \pm 0,82$ & $-3,1$ \\
SY60 & 3,44 & $3,38 \pm 0,07$ & $98,41 \pm 2,10$ & $-1,8$ \\
\hline
\end{tabular}

As amostras com maior teor de AlN em relação ao $\mathrm{Y}_{2} \mathrm{O}_{3}$, composições $\mathrm{SY} 20$, apresentaram maiores perdas de massa durante a sinterização, do que aquelas amostras cuja composição apresentam maior teor de $\mathrm{Y}_{2} \mathrm{O}_{3}$ (SY60). Este comportamento pode ser atribuído ao fato de nesta proporção de aditivos utilizado $(80 \mathrm{~mol} \%$ AlN/ $20 \mathrm{~mol} \%$ $\mathrm{Y}_{2} \mathrm{O}_{3}$ ) de acordo com o diagrama de fases do sistema AlN$\mathrm{Y}_{2} \mathrm{O}_{3}$ [12], há a formação de $\mathrm{AlN}$ + vapor (rico em AlN), que possivelmente influencia na perda de massa das amostras, devido à evaporação dos aditivos, contido na fase líquida. Nas duas composições estudadas, cerâmicas com densidade relativa próximas a 97\% foram obtidas. Baseado nesses

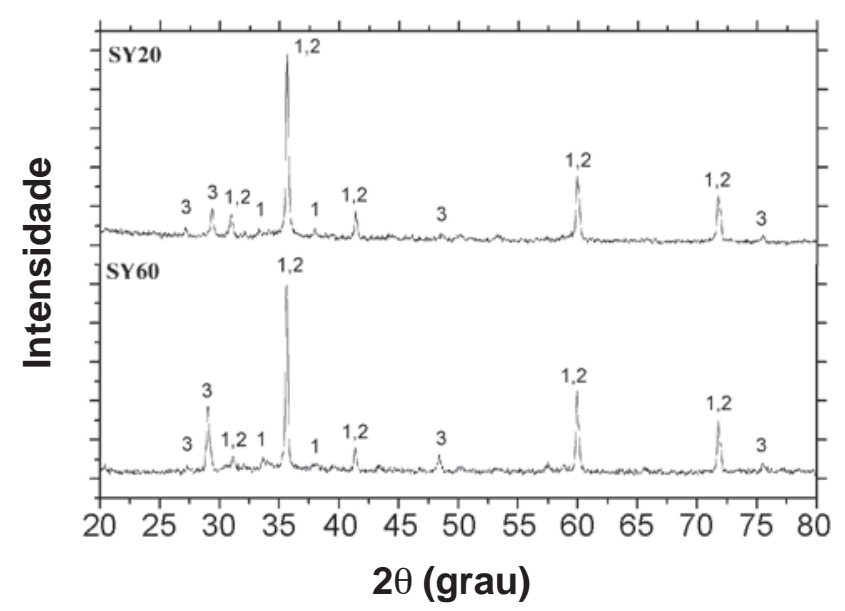

Figura 1: Difratogramas de raios $\mathrm{X}$ das amostras sinterizadas a $2080{ }^{\circ} \mathrm{C}$. (1) $\alpha$-SiC, (2) $\beta$-SiC, (3) $\mathrm{Y}_{2} \mathrm{O}_{3}$.

[Figure 1: XRD patterns of the samples sintered at $2080{ }^{\circ} \mathrm{C}$ : (1) $\alpha-\mathrm{SiC}$, (2) $\beta$-SiC, (3) $\mathrm{Y}_{2} \mathrm{O}_{3}$.] resultados, os efeitos da porosidade no comportamento a oxidação serão desconsiderados nesse estudo. Além disso, o efeito da rugosidade superficial na oxidação também será desprezado uma vez que todas as amostras sofreram preparação superficial similar.

A Fig. 1 apresenta os difratogramas de raios $\mathrm{X}$ das amostras sinterizadas.

Nota-se que há a sobreposição da fase $\alpha$-SiC à $\beta$-SiC. Estas fases, que apresentam os picos de maior intensidade são as fases majoritárias, enquanto $\mathrm{Y}_{2} \mathrm{O}_{3}$, cujos picos possuem menor intensidade, é a fase minoritária e se dissolveu parcialmente na fase intergranular amorfa formada após a sinterização. É provável que a fase AlN presente no pó de partida tenha se dissolvido totalmente nessa fase intergranular em ambas as composições estudadas, e se encontra amorfizada. Observa-se também um aumento de intensidade dos picos de $\mathrm{Y}_{2} \mathrm{O}_{3}$, proporcional a sua presença na composição global dos aditivos.
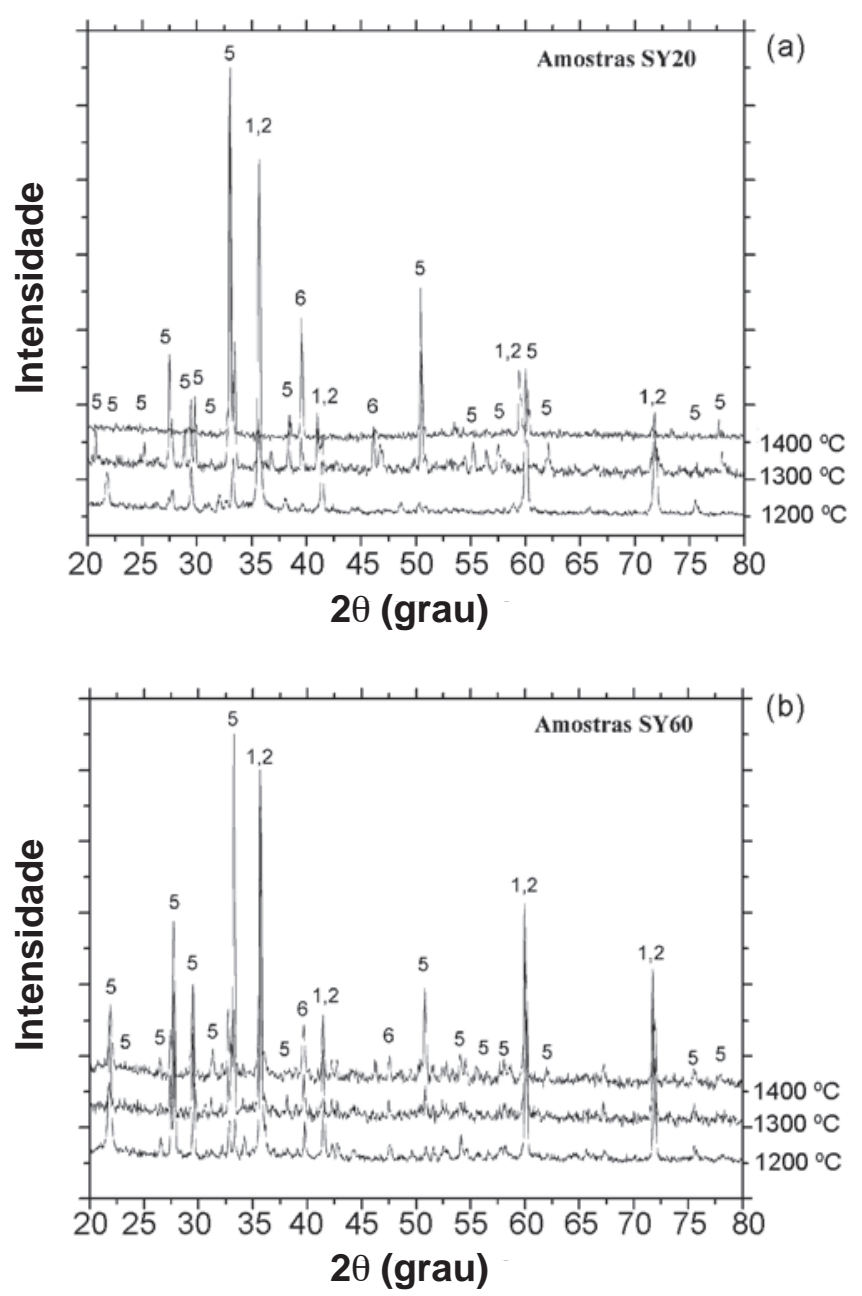

Figura 2: Evolução das fases cristalinas na superfície oxidada das amostras (a) SY20 e (b) SY60. (1) $\alpha-\mathrm{SiC}$, (2) $\beta$-SiC, (5) $\mathrm{SiO}_{2},(6)$ $\mathrm{Y}_{2} \mathrm{Si}_{2} \mathrm{O}_{7}$.

[Figure 2: Evolution of the crystalline phases on the oxidized surfaces (a) SY2O e (b) SY60. (1) $\alpha$-SiC, (2) $\beta$-SiC, (5) $\mathrm{SiO}_{2}$, (6) $\left.\mathrm{Y}_{2} \mathrm{Si}_{2} \mathrm{O}_{7^{*}}\right]$ 
A Fig. 2 mostra difratogramas de raios $X$ da superfície das amostras oxidadas após encerramento dos testes de oxidação em $120 \mathrm{~h}$, em diferentes temperaturas.

Após $120 \mathrm{~h}$ de exposição à oxidação, as principais fases cristalinas encontradas nas superfícies das amostras foram $\mathrm{SiO}_{2}$ e $\mathrm{Y}_{2} \mathrm{Si}_{2} \mathrm{O}_{7}$, presentes na camada oxidada. Estes resultados indicam que, independentemente da concentração de aditivos e da temperatura do ensaio de oxidação, estas fases foram os produtos cristalinos resultantes da oxidação e, portanto reações químicas similares podem estar ocorrendo em todas as condições de oxidação estudadas.

Sistemas SiC-AlN- $\mathrm{X}_{2} \mathrm{O}_{3}(\mathrm{X}=\mathrm{Y}, \mathrm{Er}, \mathrm{Lu}$, etc.) apresentam, após a sinterização, fases intergranulares amorfas com traços da fase cristalina $\mathrm{X}_{4} \mathrm{Si}_{2} \mathrm{~N}_{2} \mathrm{O}_{7}$, em pequena quantidade, resultante da reação entre $\mathrm{SiO}_{2}$ residual (presente na superfície do pó de $\mathrm{SiC}$ ), com os aditivos $\mathrm{AlN} \mathrm{e} \mathrm{X}_{2} \mathrm{O}_{3}$, conforme reação (B) [9-11, 13]. Assim, ao usar $\mathrm{Y}_{2} \mathrm{O}_{3}$ como aditivo, tem-se que:

$2 \mathrm{SiO}_{2(\mathrm{~s})}+2 \mathrm{Y}_{2} \mathrm{O}_{3(\mathrm{~s})}+2 \mathrm{AlN}_{(\mathrm{s})} \rightarrow \mathrm{Y}_{4} \mathrm{Si}_{2} \mathrm{~N}_{2} \mathrm{O}_{7(\mathrm{~s})}+\mathrm{Al}_{2} \mathrm{O}_{3(\mathrm{~s})}$

Não foi possível identificar as fases $\mathrm{Y}_{4} \mathrm{Si}_{2} \mathrm{~N}_{2} \mathrm{O}_{7}$ e $\mathrm{Al}_{2} \mathrm{O}_{3}$ nos difratogramas das amostras sinterizadas, conforme mostrado na Fig. 1, provavelmente devido a essas fases possuírem intensidade dos picos cristalinos abaixo do limite de detecção do difratômetro.

Durante a oxidação, a camada oxidada inicia sua formação com a reação entre o $\mathrm{SiC}$ e $\mathrm{o} \mathrm{O}_{2}$ adsorvido do ambiente através da reação $(C)$ [9-11].

$$
\mathrm{SiC}_{(\mathrm{s})}+\mathrm{O}_{2(\mathrm{~g})} \rightarrow \mathrm{SiO}_{2(\mathrm{~s})}+\mathrm{CO}_{(\mathrm{g})}
$$

Como a fase intergranular $\mathrm{Y}_{4} \mathrm{Si}_{2} \mathrm{~N}_{2} \mathrm{O}_{7}$ é incompatível com $\mathrm{SiO}_{2}$, ela decompõe-se simultaneamente ao crescimento da camada oxidada, dando origem à fase intermediária $\mathrm{Y}_{2} \mathrm{SiO}_{5}$, conforme reação (D) [9-11, 13]:

$$
2 \mathrm{Y}_{4} \mathrm{Si}_{2} \mathrm{~N}_{2} \mathrm{O}_{7(\mathrm{~s})}+3 \mathrm{O}_{2(\mathrm{~g})} \rightarrow 4 \mathrm{Y}_{2} \mathrm{SiO}_{5(\mathrm{~s})}+2 \mathrm{~N}_{2(\mathrm{~g})}
$$

A fase cristalina $\mathrm{Y}_{2} \mathrm{Si}_{2} \mathrm{O}_{7}$, observada por difração de raios $\mathrm{X}$ nas superfícies da amostras, após $120 \mathrm{~h}$ dos ensaios de oxidação é provavelmente o resultado da reação da fase $\mathrm{Y}_{2} \mathrm{SiO}_{5}$ com o $\mathrm{SiO}_{2}$, de acordo com a reação E [9-11]:

$$
\mathrm{Y}_{2} \mathrm{SiO}_{5(\mathrm{~s})}+\mathrm{SiO}_{2(\mathrm{~s})} \rightarrow \mathrm{Y}_{2} \mathrm{Si}_{2} \mathrm{O}_{7(\mathrm{~s})}
$$

De uma forma geral, observa-se, a partir dos difratogramas apresentados na Fig. 2, uma evolução da intensidade dos picos cristalinos das fases $\mathrm{SiO}_{2} \mathrm{e} \mathrm{Y}_{2} \mathrm{Si}_{2} \mathrm{O}_{7}$, em função da temperatura dos ensaios em todas as amostras, indicando que essas fases estão evoluindo e cristalizando proporcionalmente ao aumento da temperatura. Não se observa, nas superfícies das amostras oxidadas, picos de fases relativas aos aditivos utilizados ( $\mathrm{AlN}$ e $\mathrm{Y}_{2} \mathrm{O}_{3}$ ). A inexistência de picos, nas superfícies oxidadas, de fases contendo alumínio (Al) pode indicar que as mesmas se encontram presentes na forma não cristalina (amorfa) em quantidades não detectáveis por difratometria de raios $\mathrm{X}$.
Curvas de oxidação apresentando o ganho de massa por unidade de área em função do tempo de exposição, são apresentadas na Fig. 3.
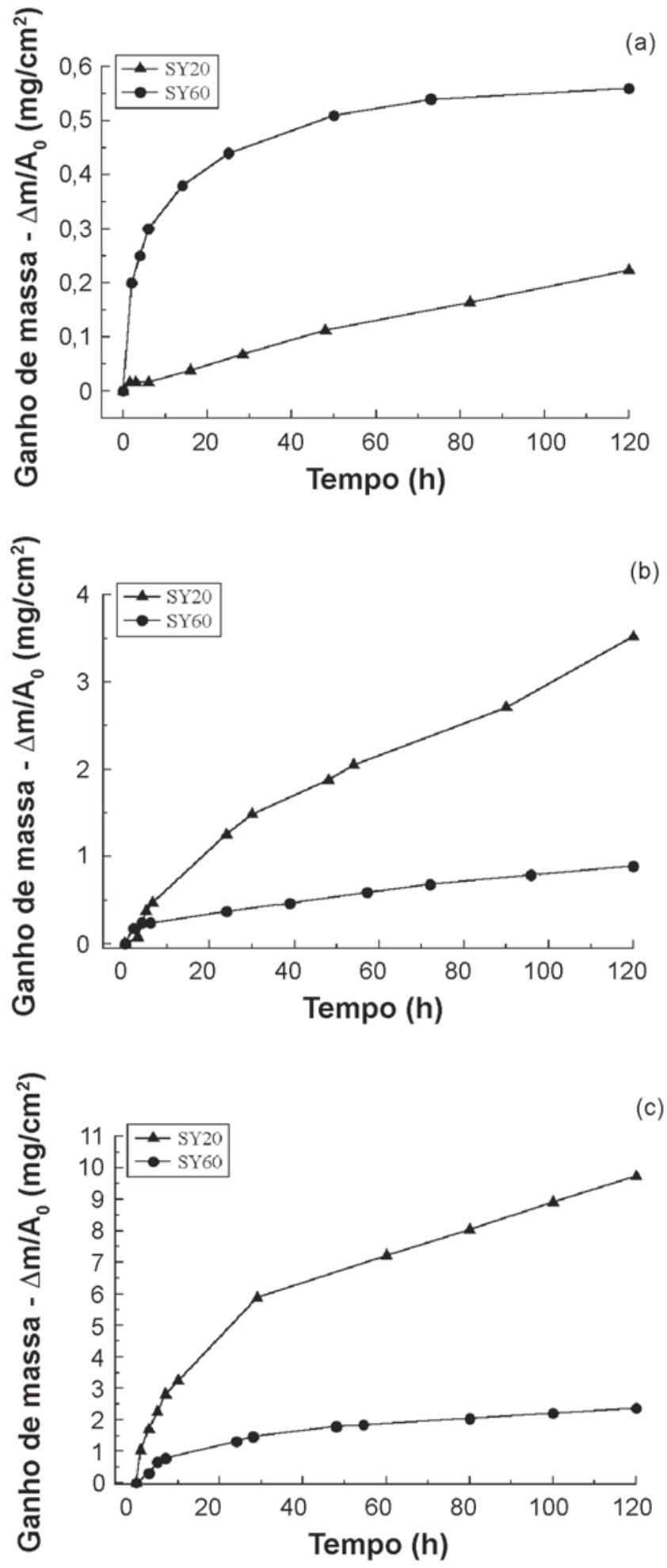

Figura 3: Curvas de oxidação apresentando o ganho de massa por unidade de área em função do tempo de exposição. (a) Ensaio a $1200{ }^{\circ} \mathrm{C}$, (b) $1300{ }^{\circ} \mathrm{C}$, (c) $1400{ }^{\circ} \mathrm{C}$.

[Figure 3: Oxidation curves presenting weight gain $x$ exposure time. (a) at $1200{ }^{\circ} \mathrm{C}$, (b) $1300{ }^{\circ} \mathrm{C}$, (c) $1400{ }^{\circ} \mathrm{C}$. ] 
Os valores de ganho de massa por unidade de área após o término dos ensaios $(120 \mathrm{~h})$ e do coeficiente de crescimento parabólico da taxa de oxidação $\left(\mathrm{k}_{\mathrm{p}}\right)$ foram calculados e estão apresentados, respectivamente, nas Tabelas III e IV.

Tabela III - Ganho de massa por unidade de área após 120 h, em função da temperatura do ensaio

[Table III - Weight gain per area unit as a function of the exposure time for different temperatures, after $120 \mathrm{~h}$.

\begin{tabular}{cccc}
\hline \multirow{2}{*}{ Amostra } & \multicolumn{3}{c}{ Ganho de massa após $120 \mathrm{~h}\left(\mathrm{mg} / \mathrm{cm}^{2}\right)$} \\
& $1200{ }^{\circ} \mathrm{C}$ & $1300{ }^{\circ} \mathrm{C}$ & $1400{ }^{\circ} \mathrm{C}$ \\
\hline SY20 & 0,22 & 3,52 & 9,73 \\
SY60 & 0,56 & 0,89 & 2,37 \\
\hline
\end{tabular}

Tabela IV - Coeficiente de crescimento parabólico da taxa de oxidação em função da temperatura do ensaio.

[Table IV - Parabolic growth coefficient of the oxidation layer as a function of temperature.]

\begin{tabular}{cccc}
\hline \multirow{2}{*}{ Amostra } & \multicolumn{3}{c}{$\mathrm{k}_{\mathrm{p}}\left(10^{-7} \mathrm{mg}^{2} \mathrm{~cm}^{-4} \mathrm{~s}^{-1}\right)$} \\
& $1200{ }^{\circ} \mathrm{C}$ & $1300{ }^{\circ} \mathrm{C}$ & $1400{ }^{\circ} \mathrm{C}$ \\
\hline SY20 & 1,1 & 271,6 & 2177,1 \\
SY60 & 2,5 & 17,8 & 130,0 \\
\hline
\end{tabular}

Comparando-se os resultados apresentados nas Tabelas III e IV, observa-se que dos materiais submetidos aos ensaios de oxidação, as amostras que apresentam menor teor de $\mathrm{Y}_{2} \mathrm{O}_{3}$ em relação ao AlN, SY20, apresentaram menor ganho de massa na temperatura de $1200^{\circ} \mathrm{C}$ e portanto menor oxidação e menor coeficiente de crescimento parabólico da taxa de oxidação $\left(\mathrm{k}_{\mathrm{p}}\right)$, enquanto as amostras que apresentam maior teor de $\mathrm{Y}_{2} \mathrm{O}_{3}$ em relação ao AlN, SY60, apresentaram maiores ganhos de massa e maiores coeficientes de crescimento parabólico da taxa de oxidação $\left(\mathrm{k}_{\mathrm{p}}\right)$, nas temperaturas de 1300 e $1400{ }^{\circ} \mathrm{C}$.

A diferença nos resultados dos ensaios de oxidação em função da variação do teor de $\mathrm{Y}_{2} \mathrm{O}_{3}$ em relação ao AlN, com a temperatura, pode estar relacionada as fases intergranulares presentes no sistema, após a sinterização [11]. Em temperaturas menores $\left(1200\right.$ e $\left.1300{ }^{\circ} \mathrm{C}\right)$, a melhor resistência à oxidação apresentada pelas amostras com menor quantidade de $\mathrm{Y}_{2} \mathrm{O}_{3}$ em relação ao AlN em seu sistema de aditivos deve-se à fase intergranular formada após a sinterização ser mais cristalina, o que torna mais difícil ao oxigênio difundir-se através da fase intergranular e assim melhorando a resistência à oxidação $[11,14]$. Por outro lado, a $1400^{\circ} \mathrm{C}$, as amostras que contém menor quantidade de $\mathrm{Y}_{2} \mathrm{O}_{3}$ em relação ao AlN em seu sistema de aditivos são menos resistentes à oxidação, provavelmente devido à presença de uma maior concentração da fase $\mathrm{Al}_{2} \mathrm{O}_{3}$ (conforme Reação B) [11, 14], que junto ao $\mathrm{SiO}_{2}$ (formado devido à oxidação de $\mathrm{SiC}$ ) formam fases de baixa viscosidade, aumentando a permeabilidade da camada óxida para o oxigênio $[11,15]$.

\section{CONCLUSÕES}

Nos ensaios de oxidação a $1200{ }^{\circ} \mathrm{C}$ as amostras contendo menor quantidade de $\mathrm{Y}_{2} \mathrm{O}_{3}$ em relação ao AlN (amostras SY20) apresentaram maior resistência a oxidação; porém, ao aumentar a temperatura para $1400{ }^{\circ} \mathrm{C}$ as amostras contendo maior quantidade de $\mathrm{Y}_{2} \mathrm{O}_{3}$ em relação ao AIN apresentaram maior resistência à oxidação. Este fato relaciona-se com as fases intergranulares do sistema, pois a $1400{ }^{\circ} \mathrm{C}$ nas amostras que apresentam menor quantidade de $\mathrm{Y}_{2} \mathrm{O}_{3}$ em relação ao AlN, possivelmente são formadas fases intergranulares de baixa viscosidade, que aumentam a permeabilidade da camada oxidada pelo oxigênio, facilitando que cátions de ítrio difundam-se para a superfície e formando maiores quantidades de óxidos.

\section{AGRADECIMENTOS}

M. J. Bondioli agradece à CAPES pela bolsa de estudos e C. dos Santos agradece à FAPESP pelo apoio financeiro fornecido para aquisição de parte da estrutura laboratorial (Proc. 04/04386-1).

\section{REFERÊNCIAS}

[1] S. Prochazka, in J. J. Burke, A. E. Gorum, R. M. Katz, (Eds.), Proc. Conf. Ceramics for High Performance Appl., Hyannis, MA, EUA, 1973. Brook Hill Publ. Co. (1975) 7-13. [2] S. Prochazka, in Special Ceramics 6, Brit. Ceram. Res. Assoc., Stoke-on-Trent (1975) 171-181.

[3] C. A. Johnson, S. Prochazka, Microstructures of sintered $\mathrm{SiC}$, in S. Fulrath (Ed.) Ceramic Microstructures 76 (1977) 366-378.

[4] M. Omori, H. Takei J. Am. Ceram. Soc. 65, 6 (1982) C-92.

[5] N. P. Padture, J. Am. Ceram. Soc. 77, 2 (1994) 519523.

[6] J.-Y. Kim, Y.-W. Kim, M. Mitomo, G.-D. Zhan, J-G. Lee J. Am. Ceram. Soc. 82, 2 (1999).

[7] M. Nader, F. Aldinger, M. J. Hoffmann J. Mater. Sci. 34 (1999) 1197-1204.

[8] G-D. Zhan, R.-J. Xie, M. Mitomo, Y.-W. Kim, J. Am. Ceram. Soc., 84, 5 (2001) 945-950.

[9] S. Guo, N. Hirosaki, H. Tanaka, Y. Yamamoto, T. Nishimura, J. Eur. Ceram.c Soc. 23 (2003) 2023-2029.

[10] H.-J. Choi, J.-G. Lee, Y.-W. Kim, J. Am. Ceram. Soc. 85, 9 (2002) 2281-2286.

[11] K. Biswas, G. Rixecker, F. Aldinger, Mater. Sci. Eng. A 374, 1-2 (2004) 56-63.

[12] A. Jeutter, Untersuchung der Phasenbeziehung im System Aluminiumnitrid-Yttriumoxid, Tese de Doutorado, Universität Stuttgart (1993).

[13] J. Costello, R. Tressler, J. Am. Ceram. Soc. 69 (1986) 674-681.

[14] R. Yue, Y. Wang, C. Chen, Surf. Sci. 148 (1999) 73-78.

[15] I. Aksay, J. Pask, J. Am. Ceram. Soc. 58, 11-12 (1975) 507-512.

(Rec. 20/08/2007, Rev. 07/11/2007, Ac. 27/12/2007) 